\title{
Effect of Opportunistic Scheduling on the Quality of Service Perceived by the Users in OFDMA Cellular Networks
}

\author{
Bartłomiej Błaszczyszyn and Mohamed Kadhem Karray
}

\begin{abstract}
Our objective is to analyze the impact of fading and opportunistic scheduling on the quality of service perceived by the users in an Orthogonal Frequenc Division Multiple Access (OFDMA) cellular network.

To this end, assuming Markovian arrivals and departures of customers that transmit some given datavolumes, as well as some temporal channel variability (fading), we study the mean throughput that the network offers to users in the long run of the system. Explicit formulas are obtained in the case of allocation policies, which may or may-not take advantage of the fading, called respectively opportunistic and nonopportunistic.
\end{abstract}

The main practical results of the present work are the following. Firstly we evaluate for the non-opportunistic allocation the degradation due to fading compared to Additive White Gaussian Noise (AWGN) (that is, a decrease of at least $13 \%$ of the throughput). Secondly, we evaluate the gain induced by the opportunistic allocation. In particular, when the traffic demand per cell exceeds some value (about $2 \mathrm{Mbits} / \mathrm{s}$ in our numerical example), the gain induced by opportunism compensates the degradation induced by fading compared to AWGN.

\footnotetext{
B. Błaszczyszyn

INRIA \& ENS, 23 Avenue d'Italie 75214 Paris Cedex 13 France

Tel.: +33-1-39635325

E-mail: Bartek.Blaszczyszyn@ens.fr

M.K. Karray

France Telecom, Research and Development Division, 38/40

rue du Général Leclerc, 92794 Issy-Moulineaux France

Tel.: +33-1-45295373

E-mail: mohamed.karray@orange-ftgroup.com
}

Keywords Radio communication, Opportunistic, Scheduling, Quality of Service, Information theory, Markov processes.

\section{Introduction}

Wireless cellular networks are in permanent and rapid evolution. They provide service for constant bit-rate (CBR) calls (such as voice calls, video conferences, etc.), and carry variable bit-rate (VBR) calls (such as mail, $\mathrm{ftp}$ ), which accept fluctuations of the rates. Customers arrive to the network and require some service. Once they are admitted, they are allocated some resource (power, bandwidth, code) for the duration of their service, then they depart from the network. Besides the dynamics due to user arrivals and departures, the channel conditions vary due to fading.

Growing traffic requires better planning and/or dimensioning of the networks. This task can be substantially simplified by analytical study of the dynamic network performance.

The objective of the present paper is to account for the effect of fading in the dynamic performance evaluation of the downlink of an Orthogonal FrequencyDivision Multiple Access (OFDMA) cellular network.

To this aim, the following two groups of elements are crucial. On one hand, the network geometry and the resource allocation, which can be chosen by the network designer. On the other hand, channel conditions and the user traffic (intensity of arrivals and requested service) can only be predicted by the designer. In order to optimize the network performance, the resource allocation should be adapted to the channel and traffic conditions. Besides better coding (up to the information theory's limit) one is looking for allocations 
that are opportunistic; i.e., take advantage of the actual channel state and user position, preserving some fairness of the mean service rates offered to users. More precisely, the opportunistic allocation assumes that at each time, each bandwidth portion is allocated to the user with the most favorable fading state. Once the resource allocation is given, the quality of service (QoS; i.e., blocking probability for CBR and throughput for VBR) perceived by the users may be evaluated using queueing theory.

Individual elements of the above puzzle (i.e. information theory, resource allocation and queueing theory) are often studied and optimized separately. The main contribution of this paper is a global approach that combines these elements to deduce the performance of the non-opportunistic and the opportunistic resource allocations. In doing so we systematically use a separation of the times scales of different elements of the network dynamics, described in Section 2.1.

The main practical results of the present work are the following. Firstly we evaluate for the non-opportunistic allocation the degradation due to fading compared to Additive White Gaussian Noise (AWGN). Secondly, we evaluate the gain induced by the opportunistic allocation.

The remaining part of this paper is organized as follows. In Section 1.1 we list these existing results in the literature that are used in our global approach. Our model of the OFDMA network is presented in Section 2. In Section 2.1 we describe the network dynamics and its decomposition into three time scales. They correspond respectively to information theory, resource allocation and queueing theory which are studied in Sections 3, 4 and 5 respectively. The numerical results are described in Section 6. Finally Section 8 describes some interesting perspectives.

\subsection{Related Work}

A growing interest in OFDMA has resulted in many publications on performance evaluation of such networks. It is not in the scope of this paper to make a thorough review of this literature. For some references on this field see for example those cited in [17]. Here we remark only those results that are directly related to our approach.

Our starting point is our work in [17] assuming AWGN channels between the users and the base stations. Coluccia et al. [11] study optimality and fairness in resource allocation. As regard to the performance of the opportunistic allocation policy over fading, we extend the approach of [6] and [19] to OFDMA networks. Moreover we use the known results for the stability and stationary distribution of the multi-class extension of the processor sharing queue; see e.g. [9,21].

The above results are used as building blocks to evaluate the dynamic performance of the non-opportunistic and the opportunistic resource allocations in OFDMA multi-cell networks. The present paper is an extended version of [5]. Besides more detailed proofs and analysis, the major extension is to show that the capacity lower bounds (which are the basis of our analytical approach) hold true even if there are a few number of interferers (Propositions 1 and 3 below). We carry this extension by building an argument based on the Jensen's inequality in place of the law of large numbers argument used in our previous work [5].

Some attempts to make other extensions of the present work are currently carried by other researchers; for example Combes et al. [12] attempt to account for frequency selective fading channels.

\section{Model assumptions}

We will consider a wireless network composed of several base stations (BS). Each BS is equipped with a single antenna (no MIMO) and its total power is limited to some given maximal value. The same frequency spectrum is available to all BS (frequency reuse factor equal to one). Each BS allocates disjoint sub-carriers to its users without macrodiversity (each user is served by exactly one BS). Thus, any given user receives only other-BS interference that is the sum of powers emitted by other BS on the sub-carriers allocated to him by his BS.

When the radio signal propagates (from a BS to a given user) it is attenuated. We model the propagationloss as the product of three factors called distance-loss, shadowing and fading. The first factor is due to the distance between the transmitter and the receiver. The shadowing is due to the attenuation by the obstacles between the transmitter and the receiver. The fading is due to the reflection on the obstacles in the neighborhood of the receiver which generate multiple paths. We account only for the distance and the fading effects in the present study.

We assume that the bandwidth of each sub-carrier is smaller than the coherence frequency of the channel, so that we can consider that the fading in each sub-carrier is flat. That is, the output of the channel at a given time depends on the input only at the same instant of time. We don't make any assumption on the correlation of the fading processes corresponding to different subcarriers (for a given user and a given BS). However, the 
fading processes for different users or base stations are assumed independent.

Time is divided into time-slots of length smaller than the coherence time of the channel, so that, for a given sub-carrier, the fading remains constant during each time-slot and the fading process in different timeslots may be assumed ergodic. (Such model for fading generalizes the so-called quasi-static model where the fading process at different time-slots is assumed to independent and identically distributed.)

The fading in each time-slot and each sub-carrier is a Rayleigh distributed random variable.

The codeword duration equals the time-slot, which is assumed sufficiently large so that the capacity within each time-slot may be defined in the asymptotic sense of the information theory.

Users perform single user detection; thus the interference is considered as additive noise and added to the additive white Gaussian noise (AWGN). The statistical properties of the interference are not known a priory since they depend of the codings of the other users. Moreover the signals transmitted by different base stations are assumed independent.

For each sub-carrier, and each time-slot, efficient (e.g. turbo) codes are used to obtain bit-rates close to the capacity given by information theory (i.e. the maximal rate for which there exist coding schemes with error probability vanishing when the length of the code is sufficiently large). In particular, for the AWGN channel the capacity equals

$C=w \log _{2}(1+\mathrm{SNR})$

where $w$ is the bandwidth and SNR designates the signal to noise power ratio.

\subsection{Separation of time scales}

We assume that there are a few time scales each associated to the evolution of some stochastic process. Roughly speaking, we assume that the duration of each time scale is sufficiently large so that the corresponding process converges to its stationary state; and sufficiently small so that the processes of the larger time scales don't evolve within this duration.

We describe now the time scales from the fastest to the slowest one.

The fastest time scale corresponds to information theory. The users number and positions as well as powers and bandwidths allocated to them are assumed fixed. Then the bit-rate of each user can not exceed the capacity given by information theory (i.e. the maximal rate for which there exist coding schemes with error probability vanishing when the length of the code is sufficiently large). This information theory constraint (i.e. existence of a coding scheme corresponding to the bitrate) together with the maximal power and bandwidth constraints constitute the resource allocation constraints.

The intermediate time scale corresponds to the resource allocation problem. The users number and positions are assumed fixed. The network attempts to allocate powers, bandwidths and bit-rates (coding schemes) to all users respecting the resource allocation constraints.

This problem may be reformulated in terms of some condition on the bit-rates. More precisely, by feasibility condition we mean a condition regarding bit-rates of different users which guarantees the existence of powers and bandwidths satisfying the resource allocation constraints.

It is the role of the load control to guarantee this condition. The load control comprises the admission control (for CBR traffic) which checks at the arrival instant of each new user the feasibility condition and decides whether it can be admitted or not; and the congestion control (for VBR traffic) which may modify the user bit-rates to satisfy the feasibility condition. Once this is done, the network allocates powers and bandwidths to all users supporting the (feasible) bit-rates.

The slowest time scale corresponds to queueing theory, where we consider the stochastic process of users positions which is driven by call arrivals and durations or data volumes. This process is subject at each time the feasibility condition described above. The stationary state (or distribution) of this process permits to calculate the quality of service perceived by the users.

\subsection{Power allocation}

The network may operate a power adaptation. Such power adaptation may be either a power control to transmit just what is necessary to compensate the interference as described in [17] or a water filling attempting to maximize the cell capacity by allocating the powers among the users according to their fadings as described in [20, Theorem 2 p.119 ].

We shall assume that the signal transmitted by each BS has a given power constant over time (which equals to the maximal authorized value) and a constant power spectral density (i.e. the power is the same for all subcarriers). The performance obtained with this assumption gives a lower bound of the performance of a network which operates a power adaptation. We shall carry our analysis by considering further lower bounds when necessary. The dimensioning based on such lower bounds is conservative. 


\subsection{Resource allocation}

We always assume that each user (receiver) knows its own fading state; a situation called CSIR (Channel State Information at Receiver) in literature.

We shall consider two resource allocation schemes:

1. Non-opportunistic: The BS doesn't take into account the fading when allocating the sub-carriers to the users. This may be due to the fact that the BS (transmitter) doesn't know the fading states of its users.

2. Opportunistic: The BS takes into account the fading when allocating the sub-carriers to the users. This requires that the BS (transmitter) knows the fading states of its users; a situation called CSIT (Channel State Information at Transmitter) in literature. The gain obtained by the opportunistic allocation is called the multiuser diversity gain.

\subsection{Possible model extensions}

Even though our model seems to have many simplifying assumptions, we will show that it permits to capture and analyze the principal impacts of fading and multiuser diversity gain in cellular OFDMA networks. Moreover the following remarks show how to extend the model.

In [15] it is observed that for OFDMA systems implementing a family of $M$-QAM modulations (as those described in [13]) with some BER target, the AWGN capacity formula (1) should be replaced by

$C=w \log _{2}\left(1+\frac{\mathrm{SNR}}{\Gamma}\right)$

where $\Gamma=-\ln (5 \times \mathrm{BER}) / 1.5$ (which is larger than 1 for $\left.\mathrm{BER}<e^{-1.5} / 5 \simeq 0.0446\right)$. Thus accounting for real coding schemes may be tentatively taken into account in our approach by an appropriate modification of the AWGN capacity formula. (For a similar question for HSDPA networks, it is observed in [16, Figure 11.1 p.175] that HSDPA coding offers a capacity which is approximately the third of the AWGN capacity.)

The assumption that the fading is constant over a time slot may be replaced by the less restrictive assumption that the fading is ergodic and perfectly known by the receiver (see [7]).

In what follows we analyze the three time scales of our model described in Section 2.1.

\section{Information theory}

We assume that the users don't move at the considered time scale. Consider a given user served by a given BS $u$. For each sub-carrier, and each time-slot, say $[0, T]$, the channel output $Y(t)$ is related to the transmitted signal $X(t)$ by

$Y(t)=S_{u} \times X(t)+Z(t)+I(t), \quad t \in[0, T]$

where $S_{u}$ represent the fading assumed constant, $Z(t)$ designate the noise assumed AWGN with power spectral density $N_{0}$ and $I(t)$ is the interference. (We skip for the moment the propagation loss induced by the distance.)

Lets denote by $p$ be the power in a given sub-carrier, that is

$E\left[|X(t)|^{2}\right]=p$

For each interfering BS $v$, let $X_{v}(t)$ be its transmitted signal and $S_{v}$ be the fading assumed constant. Then the interference equals to

$I(t)=\sum_{v \neq u} S_{v} X_{v}(t)$

Since the signals transmitted by different base stations are assumed independent (and centred),

$E\left[|I(t)|^{2}\right]=\sum_{v \neq u}\left|S_{v}\right|^{2} E\left[\left|X_{v}(t)\right|^{2}\right]=p \sum_{v \neq u}\left|S_{v}\right|^{2}$

Using [22, Theorem 18], we may show that the worst noise process distribution (not necessarily white nor Gaussian) for capacity with given second moment, is the AWGN. We deduce that the capacity $C$ within the considered time-slot is lower bounded by

$C \geq w \log _{2}\left(1+\frac{p\left|S_{u}\right|^{2}}{w N_{0}+p \sum_{v \neq u}\left|S_{v}\right|^{2}}\right)$

where $w$ is the bandwidth of a sub-carrier.

Remark 1 An alternative way to show the above inequality is to use [1, Lemma 1 p.30] giving the capacity of the interference channel. It is frequent to see in literature the equality sign in place of the inequality in the above display, as for example [20, p.118 ]. We don't see why the equality may be justified in this generality (see $[2, \S 4$ p.811] for a similar question).

We will now introduce propagation loss $L_{v}$ induced by the distance between BS $v$ and the given user. In order to account for these losses, the above formula should be replaced by

$C \geq w \log _{2}\left(1+\frac{p\left|S_{u}\right|^{2} / L_{u}}{w N_{0}+p \sum_{v \neq u}\left|S_{v}\right|^{2} / L_{v}}\right)$ 


\subsection{Ergodic capacity}

We will see soon that the expectation of the capacity with respect to the fading process (called ergodic capacity) plays a crucial role. We will not make any assumption of the distribution of the fading process except that $E\left[\left|S_{u}\right|^{2}\right]=1$

Proposition 1 The ergodic capacity $E[C]$ is lower bounded by

$E[C] \geq w E\left[\log _{2}\left(1+\beta\left|S_{u}\right|^{2}\right)\right]$

where the expectation is with respect to the fading random variables and

$\beta=\frac{p / L_{u}}{w N_{0}+p \sum_{v \neq u} 1 / L_{v}}$

which may be viewed as the signal to interference and noise ratio (SINR) in our model.

Proof Note that the expectations in the present proof are with respect to the fading random variables $S_{u}$ and $\left(S_{v}\right)_{v \neq u}$. Let $E\left[\cdot \mid S_{u}\right]$ designates the expectation conditionally to $S_{u}$. By the properties of the conditional expectation we have

$E[C]=E\left[E\left[C \mid S_{u}\right]\right]$

Equation (2) implies

$E\left[C \mid S_{u}\right] \geq w E\left[\log _{2}\left(1+\frac{\mathcal{P}\left|S_{u}\right|^{2} / L_{u}}{N+\mathcal{P} \sum_{v \neq u}\left|S_{v}\right|^{2} / L_{v}}\right) \mid S_{u}\right]$

Since the function $x \mapsto \log (1+1 / x)$ is convex on $\mathbb{R}_{+}^{*}$ (its second derivative is $\frac{2 x+1}{x^{2}(x+1)^{2}}$ ), we deduce from Jensen's inequality that the right-hand side of the above equation is larger than $E\left[\log _{2}\left(1+\beta\left|S_{u}\right|^{2}\right) \mid S_{u}\right]$ where $\beta$ is given by (3). Thus

$$
\begin{aligned}
E[C] & =E\left[E\left[C \mid S_{u}\right]\right] \\
& \geq w E\left[E\left[\log _{2}\left(1+\beta\left|S_{u}\right|^{2}\right) \mid S_{u}\right]\right] \\
& =w E\left[\log _{2}\left(1+\beta\left|S_{u}\right|^{2}\right)\right]
\end{aligned}
$$

\section{Resource allocation}

4.1 Non-opportunistic allocation

We consider a given sub-carrier and multiple time-slots. Recall that the fading for different time-slots are independent and identically distributed. By the law of large number, the capacity averaged over a large number of time-slots would approach the so-called ergodic capacity $E[C]$ where the expectation is with respect to the fading states. Proposition 1 gives a lower bound for $E[C]$.

Averaging over a large number of time-slots corresponds to exploiting the so-called time-diversity which is suitable for the analysis of the performance of $V B R$ traffic as observed in $[8, \S \mathrm{I}]$.

Remark 2 Note that the formula

$E\left[\log _{2}\left(1+\beta\left|S_{u}\right|^{2}\right)\right]$

is similar to the capacity of a channel with ergodic fading in [7] (or i.i.d. fading in [14, Equation (2)]) and CSIR (Channel State Information known at Receiver; i.e. the receiver knows the fading). Nevertheless, the fading varies during a codeword there, whereas in the model considered in the present paper the fading is constant during a codeword.

Since $\left|S_{u}\right|$ is Rayleigh distributed, i.e. it has the probability density function

$f_{\left|S_{u}\right|}(s)=2 s e^{-s^{2}} 1_{s \geq 0}$

then $H=\left|S_{u}\right|^{2}$ has exponential distribution

$f_{H}(h)=e^{-h} 1_{h \geq 0}$

In this case,

$$
\begin{aligned}
E\left[\log _{2}\left(1+\beta\left|S_{u}\right|^{2}\right)\right] & =\int_{0}^{\infty} \log _{2}(1+\beta h) e^{-h} d h \\
& =\frac{1}{\ln 2} e^{\beta^{-1}} \operatorname{Ei}\left(1, \beta^{-1}\right)
\end{aligned}
$$

where

$\operatorname{Ei}(1, x)=\int_{1}^{\infty} \frac{e^{-x t}}{t} d t$

is the exponential-integral function.

Remark 3 Frequency diversity. We consider now a given time-slot and large number $n$ of sub-carriers. Assume that the fading variables for different sub-carriers are i.i.d. (or more generally the fading process is ergodic with respect to the number $n$ of subcarriers). Then again, by the law of large number, the capacity of a large number $n$ of sub-carriers is lower bounded by

$n w E\left[\log _{2}\left(1+\beta\left|S_{u}\right|^{2}\right)\right]$

Thus the ergodic capacity is also appropriate for $C B R$ traffic (where we can't always count on time-diversity) when the number of sub-carriers allocated to each user is large enough. 


\subsubsection{Feasible bit-rates}

A base station $u$ allocates to each user $m$ in its cell (denoted with a slight abuse of notation by $m \in u$ ) a number of sub-carriers of total bandwidth $w_{m}$ (that is the bandwidth $w$ of a given sub-carrier multiplied by the number of sub-carriers allocated to the user). The following proposition gives a condition on the users bit rates $\left(r_{m}\right)_{m \in u}$ assuring the feasibility of the bandwidths allocation.

Proposition 2 Let $u$ be a given base station, $W$ be the total system bandwidth and

$\beta_{m}=\frac{p / L_{u, m}}{w N_{0}+p \sum_{v \neq u} 1 / L_{v, m}}$

be the SINR for user $m \in u$, where $N$ and $\mathcal{P}$ are respectively the noise and signal powers in a given sub-carrier (of bandwidth $w$ ) and $L_{v, m}$ is the propagation loss between BS $v$ and user $m$. If the users bit rates $\left(r_{m}\right)_{m \in u}$ satisfy the following condition

$\sum_{m \in u} \frac{r_{m}}{E\left[\log _{2}\left(1+\beta_{m}\left|S_{u}\right|^{2}\right)\right]} \leq W$

then the allocation of the bandwidths to the users is feasible.

Proof The feasibility of a bandwidth allocation may be formulated as follows. Do there exist bandwidths $\left(w_{m}\right)_{m \in u}$ whose sum doesn't not exceed the total bandwidth $W$, that is

$\sum_{m \in u} w_{m} \leq W$

We deduce from Proposition 1 that the following bitrates are feasible from the information theory point of view

$r_{m}=w_{m} E\left[\log _{2}\left(1+\beta_{m}\left|S_{u}\right|^{2}\right)\right], \quad m \in u$

where the SINR $\beta_{m}$ is given by (4). Since $\beta_{m}$ is independent of the number of sub-carriers allocated to the user, we may easily express the bandwidth as function of the bit rate as follows

$w_{m}=\frac{r_{m}}{E\left[\log _{2}\left(1+\beta_{m}\left|S_{u}\right|^{2}\right)\right]}$

The condition (5) on the bit-rates assures that the sum of the bandwidths given by the above equation doesn't not exceed the total bandwidth $W$.
Note that (5) may be written as follows

$\sum_{m \in u} r_{m} \gamma_{m} \leq 1$

where

$\gamma_{m}=\left[W E\left[\log _{2}\left(1+\beta_{m}\left|S_{u}\right|^{2}\right)\right]\right]^{-1}$

Each bit-rate allocation satisfying (6) will be called non-opportunistic allocation. Note that the constraint (6) on the bit-rates do not depend on the current values of the fadings but only on their statistics.

\subsection{Opportunistic allocation}

We assume that the BS knows the fading states of all the users in its cell and that each user knows his own fading state.

\subsubsection{Analysis for a given subcarrier}

We account now for the fading variations at the resource allocation time scale. In what follows, by opportunistic allocation we mean that at a given time-slot, each subcarrier is allocated to the user with the most favorable fading state; more precisely, each BS $u$ allocates the subcarrier to the user $m$ such that

$\left|S_{u, m}\right|=\max _{n \in u}\left|S_{u, n}\right|$

We aim now to calculate the ergodic throughput of each user per time-slot under this policy.

If we allocate a given subcarrier to a user $m \in u$, then we deduce from (2) that, for a given time-slot, we can find a coding scheme to support the following bit-rate

$R_{m}=w \log _{2}\left(1+\frac{p\left|S_{u, m}\right|^{2} / L_{u, m}}{N+p \sum_{v \neq u}\left|S_{v, m}\right|^{2} / L_{v, m}}\right), \quad m \in u$

where the $S_{v, m}$ are the fading states between the user $m$ and BS $v$ for the considered subcarrier and time-slot. Let us denote by $\bar{R}_{m}$ the bit-rate effectively allocated by the opportunistic policy to a given user $m$ averaged over a large number of time-slots. From the assumed temporal ergodicity, we deduce that

$\bar{R}_{m}=E\left[R_{m} \times 1\left\{\left|S_{u, m}\right|=\max _{n \in u}\left|S_{u, n}\right|\right\}\right]$

Proposition 3 Assume that for a given time-slot the bit-rate of user $m$ is given by

$R_{m}=w \phi\left(\frac{p\left|S_{u, m}\right|^{2} / L_{u, m}}{N+p \sum_{v \neq u}\left|S_{v, m}\right|^{2} / L_{v, m}}\right), \quad m \in u$ 
where $\phi$ is a function defined on $\mathbb{R}_{+}$having a well defined Laplace transform $\mathcal{L}_{\phi}(u)=\int_{0}^{+\infty} \phi(x) e^{-u x} d x$; and such that the function $x \mapsto \phi(1 / x)$ is convex on $\mathbb{R}_{+}$. Then for the opportunistic allocation (8), the bitrate effectively allocated to user $m$ and averaged over a large number of time-slots is lower bounded by

$$
\begin{aligned}
\bar{R}_{m} & \geq R_{m}^{\prime} \\
& :=w E\left[\phi\left(\beta_{m}\left|S_{u, m}\right|^{2}\right) \times 1\left\{\left|S_{u, m}\right|=\max _{n \in u}\left|S_{u, n}\right|\right\}\right] \\
& =w \int_{0}^{+\infty} \phi\left(\beta_{m} x\right) e^{-x}\left(1-e^{-x}\right)^{M-1} d x \\
& =\frac{w}{\beta_{m}} \sum_{k=0}^{M-1}\left(\begin{array}{c}
M-1 \\
k
\end{array}\right)(-1)^{k} \mathcal{L}_{\phi}\left(\frac{k+1}{\beta_{m}}\right)
\end{aligned}
$$

where $\beta_{m}$ is given by (4) and $M$ is the number of the users in the cell.

Proof Since the function $x \mapsto \log (1+1 / x)$ is convex, the inequality (10) follows from Jensen's inequality. Since the $\left|S_{u, n}\right|^{2}$ are exponentially distributed, we get

$R_{m}^{\prime}$

$=w \int_{\mathbb{R}_{+}^{M}} \phi\left(\beta_{m} x_{m}\right) 1\left\{x_{m}=\max _{n \in u} x_{n}\right\} \prod_{n \in u} e^{-x_{n}} d x_{n}$

$=w \int_{\mathbb{R}_{+}} \phi\left(\beta_{m} x\right) e^{-x} \int_{\mathbb{R}_{+}^{M-1}} \prod_{n \neq m} 1\left\{x_{n} \leq x\right\} e^{-x_{n}} d x_{n} d x$

$=w \int_{\mathbb{R}_{+}} \phi\left(\beta_{m} x\right) e^{-x} \prod_{n \neq m} \int_{\mathbb{R}_{+}} 1\left\{x_{n} \leq x\right\} e^{-x_{n}} d x_{n} d x$

$=w \int_{\mathbb{R}_{+}} \phi\left(\beta_{m} x\right) e^{-x}\left(1-e^{-x}\right)^{M-1} d x$

$=w \int_{0}^{+\infty} \phi\left(\beta_{m} x\right) e^{-x}\left(1-e^{-x}\right)^{M-1} d x$

Using the binomial formula

$\left(1-e^{-x}\right)^{M-1}=\sum_{k=0}^{M-1}\left(\begin{array}{c}M-1 \\ k\end{array}\right)(-1)^{k} e^{-k x}$

we get

$$
\begin{aligned}
R_{m}^{\prime} & =w \sum_{k=0}^{M-1}\left(\begin{array}{c}
M-1 \\
k
\end{array}\right)(-1)^{k} \int_{0}^{+\infty} \phi\left(\beta_{m} x\right) e^{-(k+1) x} d x \\
& =w \sum_{k=0}^{M-1}\left(\begin{array}{c}
M-1 \\
k
\end{array}\right)(-1)^{k} \int_{0}^{+\infty} \phi(y) e^{-\frac{k+1}{\beta_{m}} y} \frac{d y}{\beta_{m}} \\
& =\frac{w}{\beta_{m}} \sum_{k=0}^{M-1}\left(\begin{array}{c}
M-1 \\
k
\end{array}\right)(-1)^{k} \mathcal{L}_{\phi}\left(\frac{k+1}{\beta_{m}}\right)
\end{aligned}
$$

We prove now that the Shannon function $\log _{2}(1+x)$ satisfies the conditions of the above proposition.
Lemma 1 The function $\phi(x)=\log _{2}(1+x)$ has a well defined Laplace transform given by

$\mathcal{L}_{\phi}(u)=\frac{e^{u}}{u \ln 2} \operatorname{Ei}(1, u)$

and the function $x \mapsto \phi(1 / x)$ is convex on $\mathbb{R}_{+}$.

Proof The Laplace transform of $\phi(x)=\log _{2}(1+x)$ is

$$
\begin{aligned}
\mathcal{L}_{\phi}(u) & =\int_{0}^{+\infty} \log _{2}(1+x) e^{-u x} d x \\
& =\frac{1}{\ln 2} \int_{0}^{+\infty} \ln (1+x) e^{-u x} d x \\
& =\frac{1}{\ln 2} \int_{0}^{+\infty} \frac{e^{-u x}}{u(1+x)} d x \\
& =\frac{e^{u}}{u \ln 2} \int_{1}^{\infty} \frac{e^{-u t}}{t} d t=\frac{e^{u}}{u \ln 2} \operatorname{Ei}(1, u)
\end{aligned}
$$

where for the third line we use an integration by parts. The convexity of the function $x \mapsto \phi(1 / x)$ on $\mathbb{R}_{+}$was already shown in the proof of Proposition 1.

\subsubsection{Symmetric case}

The expression of $R_{m}^{\prime}$ (defined by (10)) in the case when $\phi$ is linear; that is $\phi(x)=x / \ln 2$ was already given by Borst $[6, \S \mathrm{II}]$ who showed that

$R_{m}^{\prime}=\left(\frac{1}{M} \sum_{k=1}^{M} \frac{1}{k}\right) \frac{w \beta_{m}}{\ln 2}$

This case is called symmetric in $[6, \S \mathrm{II}]$ since the random variables $\phi\left(\beta_{m}\left|S_{u, m}\right|^{2}\right)$ normalized by their expectations with respect to the fading states are identically distributed.

On the other hand, $\mathcal{L}_{\phi}(u)=\frac{1}{u^{2} \ln 2}$, thus we deduce from (12) that

$R_{m}^{\prime}=\left(\sum_{k=0}^{M-1}\left(\begin{array}{c}M-1 \\ k\end{array}\right)(-1)^{k} \frac{1}{(k+1)^{2}}\right) \frac{w \beta_{m}}{\ln 2}$

which is coherent with Equation (14) since it is straightforward to prove that

$\sum_{k=0}^{M-1}\left(\begin{array}{c}M-1 \\ k\end{array}\right)(-1)^{k} \frac{1}{(k+1)^{2}}=\frac{1}{M} \sum_{k=1}^{M} \frac{1}{k}$

(Indeed, letting $f(x)=\sum_{k=0}^{M-1}\left(\begin{array}{c}M-1 \\ k\end{array}\right)(-1)^{k} \frac{e^{-(k+1) x}}{(k+1)^{2}}$ and observing that $f^{\prime \prime}(x)=e^{-x}\left(1-e^{-x}\right)^{M-1}$ we get $f(x)=$ $\frac{1}{M} \sum_{k=1}^{M} \frac{1-\left(1-e^{-x}\right)^{k}}{k}$ which applied to $x=0$ gives the desired result.)

Thus the expression of $R_{m}^{\prime}$ in our Proposition 3 may be seen as an extension of the result in $[6, \S I I]$ to the 
non-symmetric case; since it holds true even when the random variables $\phi\left(\beta_{m}\left|S_{u, m}\right|^{2}\right)$ normalized by their expectations are not necessarily identically distributed.

Note finally that Equation (14) may be written as follows

$R_{m}^{\prime}=\frac{1}{h(M)} \frac{w}{W} \frac{1}{\gamma_{m}^{\prime}}$

where $h(M)=M / \sum_{k=1}^{M} 1 / k$ and $\gamma_{m}^{\prime}=\frac{\ln 2}{W \beta_{m}}$. Thus, in the symmetric case, $R_{m}^{\prime}$ is decomposed into some function the number $M$ of the users multiplied by some function of the position of user $m$. This particular form allows an explicit calculus of the performance at the queueing theory time-scale as will be shown in Proposition 5 below.

\subsubsection{Feasible bit-rates}

The bit-rate for user $m$ over all the bandwidth, say $r_{m}^{\prime}$, is related to the bit-rate $R_{m}^{\prime}$ per sub-carrier by

$r_{m}^{\prime}=\frac{W}{w} R_{m}^{\prime}$

where $R_{m}^{\prime}$ is given by (11).

Example 1 In the particular case when $R_{m}^{\prime}$ is given by (15), then

$r_{m}^{\prime}=\frac{1}{h(M)} \frac{1}{\gamma_{m}^{\prime}}$

\section{Queueing theory}

\subsection{Traffic dynamics}

Denote the geographic region covered by the a cell by $\mathbb{D}$ that is assumed to be a bounded subset of the plane $\mathbb{R}^{2}$. Consider only VBR calls whose inter-arrival times to $\mathbb{D}$ are independent and identically distributed (i.i.d.) exponential random variables with rate $\lambda$ (mean $1 / \lambda)$. The position of each arrival is picked at random in $\mathbb{D}$ according to the uniform distribution. We assume that users don't move during their calls. Each call requires to transmit a given volume of data (amount of bits that has to be sent or received), which is modeled by an independent of everything else, exponential random variable with parameter $\mu$. The quantity $\rho=\lambda / \mu$ is called the traffic demand (expressed in $\mathrm{Mbps}^{1}$ ) per cell. Users are served by the BS according to some bit-rate allocation policy.

The set of positions of all users served at a given time is called configuration of users. Let $\mathbb{M}$ be the set

1 The abbreviation Mbps designates "Kilo-bit per second". of all possible configurations (this can be formalized e.g. on the basis of the theory of point processes). We denote by $\left\{N_{t}\right\}_{t \geq 0}$ the process describing the evolution in time of the user configurations in $\mathbb{D}$ (due to arrivals and departures). It takes its values in $\mathbb{M}$. If the process $\left\{N_{t}\right\}_{t>0}$ isn't ergodic, then the mean number of users in the system grows unboundedly in the long run of the system. This situation has to be avoided; in which case we say that the system is stable (or equivalently ergodic).

One distinguish two milestones of the analytical evaluation of the network performance: identification of its stability region, and the evaluation of the steady state characteristics (e.g. the mean throughput and delay).

\subsection{Non-opportunistic allocation}

Define the critical traffic demand $\rho_{c}$ by

$\rho_{c}=\frac{1}{\bar{\gamma}}$

where $\bar{\gamma}$ is the expectation of $\gamma_{m}$ (which is given by (7)) with respect to the position of the user $m$ uniformly distributed in the cell.

Proposition 4 Assume any non-opportunistic workconserving bit-rate allocation satisfying Condition (6). If the traffic demand per cell

$\rho<\rho_{c}$

then the system is stable. Consider the particular allocation

$r_{m}=\frac{1}{M \gamma_{m}}$

Then, at the steady state, the expected number of users in cell $u$, denoted $\bar{N}$, equals

$\bar{N}=\frac{\rho}{\rho_{c}-\rho}$

the mean delay per call, denoted $\bar{T}$, is

$\bar{T}=\frac{1}{\mu \bar{r}}=\frac{1}{\mu\left(\rho_{c}-\rho\right)}$

and the mean throughput, denoted $\bar{r}$, equals

$\bar{r}=\rho_{c}-\rho$

Proof A bit-rate allocation is said to be work-conserving when $\sum_{m \in u} r_{m} \gamma(m)=1$ as long as there is at least a user in the cell. The stability condition (18) and the expression (20) of the users number at the steady state follow from known results for multi-class processor sharing queues [10,18]. Applying Little's formula [3] we get 
the expression (21) for the delay. Using the fact that the throughput $\bar{r}$ is the ratio of the date volume average , which equals $1 / \mu$, and the delay $\bar{T}$ proves Equation (22).

\subsection{Opportunistic allocation}

The following proposition gives the performance of the opportunistic allocation (17).

Proposition 5 Consider the opportunistic bit-rate allocation (17) and denote by $\bar{\gamma}^{\prime}$ the expectation of $\gamma_{m}^{\prime}$ with respect to the user's location $m$ uniformly distributed in the cell. If the traffic demand per cell

$\rho<\frac{1}{\bar{\gamma}^{\prime}} \lim _{n \rightarrow \infty} \frac{n}{h(n)}$

then the system is stable. Moreover, at the steady state, the mean throughput per user, denoted $\bar{r}^{\prime}$, equals

$\bar{r}^{\prime}=\frac{1}{\bar{\gamma}^{\prime}} \frac{1}{\mathcal{H}\left(\bar{\gamma}^{\prime} \rho\right)}$

where the function $\mathcal{H}(s)$ is defined for $s>0$ by

$\mathcal{H}(s)=\frac{E[H(X+1)]}{E[H(X)]}, \quad H(M)= \begin{cases}\prod_{k=1}^{M} h(k) & \text { if } M \geq 1 \\ 1 & \text { if } M=0\end{cases}$

where $X$ is a Poisson random variable with parameter s. Moreover, the mean delay is

$\bar{T}=\frac{1}{\mu \bar{r}^{\prime}}=\frac{\bar{\gamma}^{\prime} \mathcal{H}\left(\bar{\gamma}^{\prime} \rho\right)}{\mu}$

and the mean number of users at the steady state equals $\bar{\gamma}^{\prime} \rho \mathcal{H}\left(\bar{\gamma}^{\prime} \rho\right)$

Proof See [6, Proposition 3.1] and [16, §11.1].

\subsubsection{Approximation}

Note that Proposition 5 assumes a particular case when the decomposition (17) holds true as in the symmetric case described in Section 4.2.2.

Unfortunately, for the more general allocation (16) we do not know a closed form for the performance; especially because the geometric parameters $\beta_{m}$ depend of the location of the user $m$. The idea is then to replace these parameters by a constant $\beta_{0}$ calculated as follows.

$\beta_{0}$ is determined in such a way that the average over the cell of the factors $\gamma_{m}$ given by Equation (7) should remain unchanged when the $\beta_{m}$ are replaced by $\beta_{0}$. (By Proposition 4, this is equivalent to say that the performance of the non-opportunistic allocation (19) remains unchanged when the $\beta_{m}$ are replaced by $\beta_{0}$.)

We will approximate the performance of our opportunistic scheme (16) by replacing $\beta_{m}$ with $\beta_{0}$ and applying Proposition 5.

\section{Numerical results}

\subsection{Model specification}

In order to obtain numerical values, we consider the most popular hexagonal network model, where the base stations are placed on a regular infinite hexagonal grid. Let $R$ be the radius of the disc whose area is equal to that of the hexagonal cell served by each base station, and call $R$ the cell radius. We take $R=0.525 \mathrm{~km}$. We assume a distance-loss $L(r)=(K r)^{\eta}$, with $\eta=3.38$, $K=8667 \mathrm{~km}^{-1}$. This means that the distance-loss between BS $u$ and user $m$ is equal to $L\left(\left|x_{u}, x_{m}\right|\right)$ where $x_{u}, x_{m}$ denote, respectively, the geometric location of $u$ and $m$ and $|\cdot|$ is the Euclidean distance.

We consider VBR traffic with traffic demand assumed (spatially) uniform over the cell. We consider a traffic demand per cell $\rho$ varying from 0 to $20 \mathrm{Mbps}$.

The BS maximal total power is $\tilde{P}=52 \mathrm{dBm}$ (accounting for antenna gains and losses), the total bandwidth is $W=5 \mathrm{MHz}$ and the ambient noise power $W N_{0}=-103 \mathrm{dBm}$.

\subsection{Results}

Figure 1 shows the throughput per user as function of the traffic demand per cell for the opportunistic allocation. We show the result of the simulations and of the approximation described in Section 5.3.1. The two curves are close which validates the considered approximation.

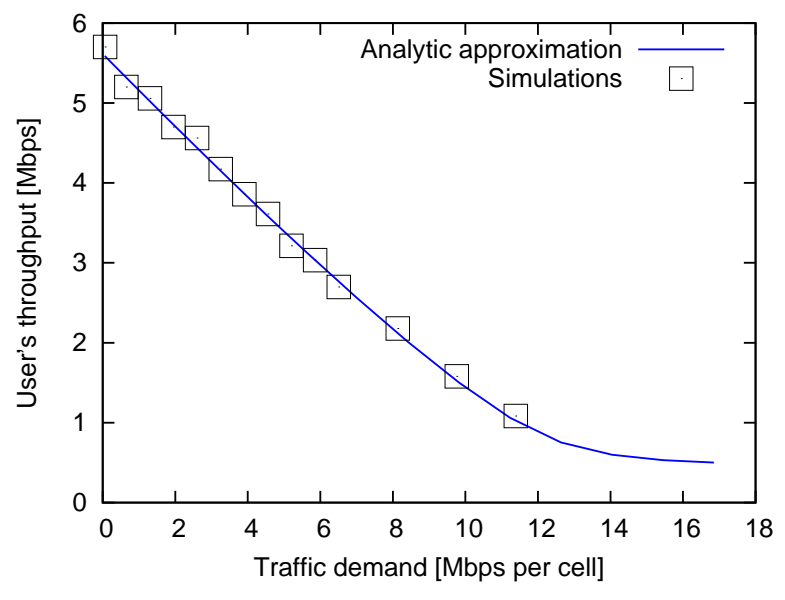

Fig. 1 Validation of the approximation for the opportunistic allocation.

Figure 2 shows the throughput per user as function of the traffic demand per cell for the AWGN, nonopportunistic and opportunistic allocation. Firstly, we 
observe that for the non-opportunistic allocation, the fading decreases the throughput per user of at least $13 \%$ compared to AWGN. Note moreover that the nonopportunistic allocation gives, like AWGN, the throughput that is a linear function of the demanded traffic in the bounded stability region. Secondly, the gain induced by opportunism increases with the traffic. When the traffic demand is very small, the gain induced by the opportunistic allocation is negligible. Nevertheless, when the traffic demand per cell exceeds some value (about $2 \mathrm{Mbps}$ in our example) the gain induced by opportunism compensates the degradation induced by fading compared to AWGN.

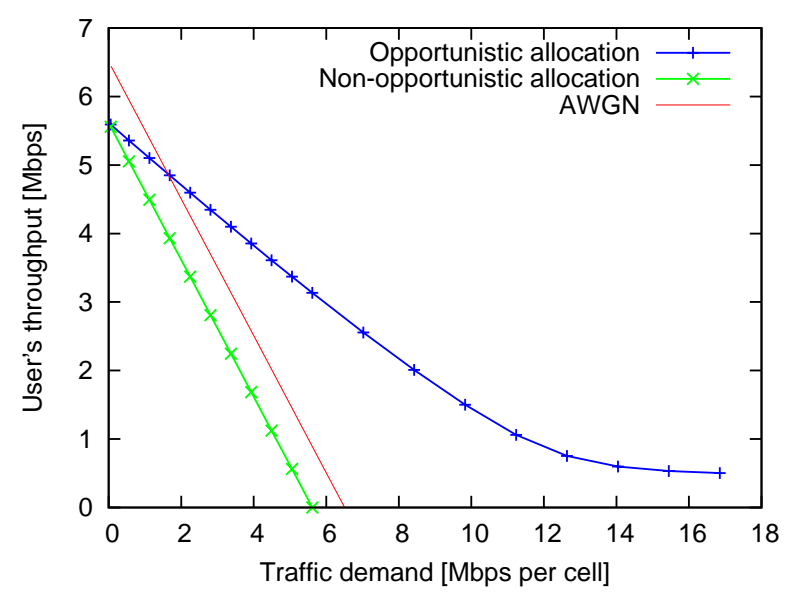

Fig. 2 Comparison of the perfomance of the different allocations.

\section{Conclusion}

We have given analytic expressions for the mean throughput and delay in OFDMA cellular networks with VBR traffic. These formulas take into account in a simple but not simplistic way all important elements of the network performance: the network geometry, the traffic dynamics, fading and bit-rate allocation policy. They allow, in particular, to compare in a systematic way the effect on performance of fading for either non-opportunistic or opportunistic allocation policies.

\section{Perspectives}

Even though we assumed Rayleigh distribution for the fading, the approach may be extended to other distributions such as Rice, Nakagami, etc.
It may be useful to study the effect of grouping the sub-carriers into the so-called chunks (i.e. a group of sub-carriers and a number of time-slots); see [20, §6.2].

As observed in $[20, \S 6.2]$, the non-opportunistic allocation scheme is suitable for fast varying fading, due to high mobility of users for example, which makes difficult to track of the rapidly varying channel. On the other hand, the opportunistic allocation scheme is suitable for slow varying fading as in the case of fixed (or pedestrian) users for example. Thus, from the point of view of the present paper, mobility decreases performance. It is interesting to couple this with the account of the speed effect at the queueing theory time scale in [4] which have shown that mobility ameliorates performance.

\section{Acknowledgments}

We thank F. Baccelli at INRIA for motivating discussions on resource allocation and queueing theory. We thank also M. Debbah from Alcatel-Lucent for instructive exchanges about information theory.

\section{References}

1. R. Ahlswede. Multi-way communication channels. In Proc. 2nd. Int. Symp. Information Theory (Tsahkadsor, Armenian S.S.R.), pages 23-52. Publishing House of the Hungarian Academy of Sciences, 1971.

2. R. Ahlswede. The capacity region of a channel with two senders and two receivers. Ann. Prob., 2:805-814, 1974.

3. F. Baccelli and P. Brémaud. Elements of queueing theory. Palm martingale calculus and stochastic recurrences. Springer, 2003.

4. B. Błaszczyszyn and M. K. Karray. Performance evaluation of scalable congestion control schemes for elastic traffic in cellular networks with power control. In Proc. of IEEE Infocom, pages 170-178, May 2007.

5. B. Błaszczyszyn and M. K. Karray. Fading effect on the dynamic performance evaluation of ofdma cellular networks. In Proc. of ComNet, November 2009.

6. S. Borst. User-level performance of channel-aware scheduling algorithms in wireless data networks. In Proc. of IEEE Infocom, 2003.

7. G. Caire and S. Shamai. On the capacity of some channels with channel state information. IEEE Trans. Inf. Theory, 45:2007-2019, September 1999.

8. G. Caire, G. Taricco, and E. Biglieri. Optimum power control over fading channels. IEEE Trans. Inf. Theory, 45(5):1468-1489, July 1999.

9. J. W. Cohen. On regenerative processes in queueing theory. Springer Verlag, 1976.

10. J. W. Cohen. The multiple phase service network with generalized processor sharing. Acta Informatica, 12:245$284,1979$.

11. A. Coluccia, A. D'Alconzo, and F. Ricciato. On the optimality of max-min fairness in resource allocation. Annals of Telecommunications, February 2011. 
12. R. Combes, Z. Altman, and E. Altman. Scheduling gain for frequency-selective rayleigh-fading channels with application to self-organizing packet scheduling. Performance Evaluation, In Press, Corrected Proof, 2011.

13. A. J. Goldsmith and S.-G Chua. Variable-rate variablepower MQAM for fading channels. IEEE Trans. Commun., 45:1218-1230, 1997.

14. A.J. Goldsmith and P. Varaiya. Capacity of fading channels with channel side information. IEEE Trans. Inf. Theory, 43:1986-1992, November 1997.

15. J. Jang and K. B. Lee. Transmit power adaptation for multiuser OFDM systems. IEEE J. Select. Areas Commun., 21(2), February 2003.

16. M. K. Karray. Analytic evaluation of wireless cellular networks performance by a spatial Markov process accounting for their geometry, dynamics and control schemes. $\mathrm{PhD}$ thesis, Ecole Nationale Supérieure des Télécommunications, 2007.

17. M. K. Karray. Analytical evaluation of QoS in the downlink of OFDMA wireless cellular networks serving streaming and elastic traffic. IEEE Trans. Wireless Commun., 9(5), May 2010.

18. L. Kleinrock. Queueing systems. Wiley and Sons, 1976.

19. H. J. Kushner and P. A. Whiting. Convergence of proportional-fair sharing algorithms under general conditions. IEEE Trans. Wireless Commun., 3(4), July 2004.

20. H. Liu and G. Li. OFDM-based broadband wireless networks: Design and optimization. Wiley-Interscience, 2005.

21. R. Serfozo. Introduction to stochastic networks. Springer, New York, 1999.

22. C. Shannon. A mathematical theory of communication. Bell Sys. Tech. J., 27:379-423, 623-656, 1948. 